\title{
Simulation and Measurements of VSWR for Microwave Communication Systems
}

\author{
Bexhet Kamo, Shkelzen Cakaj, Vladi Koliçi, Erida Mulla \\ Faculty of Information Technology, Polytechnic University of Tirana, Tirana, Albania \\ Email: bkamo@fti.edu.al, shkelzen.cakaj@fulbrightmail.org,vkolici@fti.edu.al,emulla@fti.edu.al
}

Received September 5, 2012; revised October 11, 2012; accepted October 18, 2012

\begin{abstract}
Nowadays, microwave frequency systems, in many applications are used. Regardless of the application, all microwave communication systems are faced with transmission line matching problem, related to the load or impedance connected to them. The mismatching of microwave lines with the load connected to them generates reflected waves. Mismatching is identified by a parameter known as VSWR (Voltage Standing Wave Ratio). VSWR is a crucial parameter on determining the efficiency of microwave systems. In medical application VSWR gets a specific importance. The presence of reflected waves can lead to the wrong measurement information, consequently a wrong diagnostic result interpretation applied to a specific patient. For this reason, specifically in medical applications, it is important to minimize the reflected waves, or control the VSWR value with the high accuracy level. In this paper, the transmission line under different matching conditions is simulated and experimented. Through simulation and experimental measurements, the VSWR for each case of connected line with the respective load is calculated and measured. Further elements either with impact or not on the VSWR value are identified. Interpretation of simulation and experimental results allows to judge about improving the VSWR, and consequently increasing the microwave transmission systems efficiency.
\end{abstract}

Keywords: Microwave; Load; Impedance Matching; VSWR

\section{Introduction}

Generally, when a transmitter is connected through a transmission line to an antenna, or any other load connected to, these elements must match to each other, in order to enable the maximum possible energy transfer from the transmission line to the antenna or the load, and consequently having minimal losses. When the antenna or load and transmission line that connects the transmitter from one side, and the antenna or the load to the other side, are not matched, energy is not transmitted properly. A part of energy that comes from transmitter does not go to the antenna or the load but it is reflected back, to the transmitter. So, a part of the energy that comes from the incident wave it is transmitted toward antenna, or any other load connected to the line, but the other part of it in form of waves is reflected back. Due to the presence of those waves, in the transmission line, a standing wave is created. In microwave radio planning, it is necessary to measure the voltage standing wave ratio in order to understand the mismatch level in the transmission line.

The power reflected back to the transmitter affects the performance of RF transmitter [1-4]. Standing waves are determined by the ratio of the maximum and minimum voltage amplitude of the wave in transmission line, so called VSWR (Voltage Standing Wave Ratio). In the second section, the theoretical VSWR concepts are given, and further the simulation and experiments are described with respective result analysis and conclusions.

\section{VSWR Concepts}

Generally, a microwave communication system consists of three main parts, [1-4]:

- Radio transmitter.

- Radio receiver.

- Link/wireless channel between two antennas.

The main elements of the transmitter are: oscillator, modulator, amplifier and antenna. On other hand the main elements of the receiver are: antenna, low noise amplifier, selective filter, local oscillator, mixer, intermediate frequency amplifier, and demodulator that gives at the output the signal to be received. All elements in transmitter or in receiver part are connected with each other using transmission lines. Thus, it is too important to assess electromagnetic waves transport over these transmission lines and what energy is reflected back due to a mismatch [1-4]. For further analysis it is considered a transmission line with impedance $Z_{0}$, which is connected with a load $Z_{L}$ as in Figure 1. 


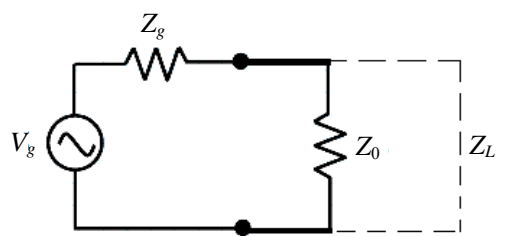

Figure 1. A transmission line with $Z_{0}$ impedance connected with a load $Z_{L}$.

If $Z_{L}$ differs from $Z_{0}$, we have a mismatch between load and line. In this case, a part of energy goes to the load and a part of it is reflected back through line to the generator $V_{g}$. The reflection coefficient along the line is defined as [1-4]:

$$
p=\frac{Z-Z_{0}}{Z+Z_{0}}
$$

or, expressed by voltage levels, the reflection coefficient is defined as the ratio of the reflected voltage $\left(V_{r}\right)$ to the incident voltage $\left(V_{i}\right)$, as:

$$
p=\frac{V_{r}}{V_{i}}
$$

The incident wave and reflected wave create the so called steady wave at transmission line. The steady wave is taken as the sum of the downward wave that passes along the line to the load and reflected wave that comes back. VSWR is defined as the ratio between maximum and minimum values of the steady wave as [1-4]:

$$
\operatorname{VSWR}=\frac{\left|V_{\max }\right|}{\left|V_{\min }\right|}
$$

If the incident and reflected voltage are in phase, these ads up, creating maximum voltage value, as:

$$
\left|V_{\max }\right|=\left|V_{i}\right|+\left|V_{r}\right|
$$

where $V_{i}$ is the r.m.s (route mean square) value of incident voltage and $V_{r}$ is the r.m.s value of the reflected voltage. Also,

$$
\left|V_{\text {min }}\right|=\left|V_{i}\right|-\left|V_{r}\right|
$$

From VSWR and reflection coefficient definition yields out the correlation between them as follows:

$$
\operatorname{VSWR}=\frac{(1+|p|)}{(1-|p|)}
$$

In case the line is matched with the load, $Z_{L}=Z_{0}$ then the reflection factor is $p=0 \quad\left(V_{r}=0\right)$, so VSWR $=1$. This is the best scenario when the line with load it is perfectly matched [5].

\section{VSWR Simulation}

Considering the importance of VSWR for communica- tion systems, the impedance mismatch of microwave waveguide when connected to a specific load through simulation is analyzed. The load may be too close to the characteristic impedance of a waveguide as the best case, or it might be too different compared to specific impedance as the worst case [6]. For simulation purposes three scenarios are considered. The first scenario considers a gateway, a transmission line and a load, as presented in Figure 2.

In this case the transmission line sizes are fixed and the load is the varying parameter. The length of the line is $69 \mathrm{~mm}$, characteristic impedance $Z_{0}=50 \mathrm{ohm}$ and the load changes from $50 \mathrm{ohm}$ to $200 \mathrm{ohm}$. Figures 2(a) and (b) respectively show the cases for $50 \mathrm{ohm}$ and $100 \mathrm{ohm}$. Simulation is performed at frequency of $3 \mathrm{GHz}$. Under the load of $50 \mathrm{ohm}$, the same as the characteristic impedance $Z_{0}$ of the line, the VSWR, is equal to 1 , as shown by simulation at Figure 2(a). If the load changes to $100 \mathrm{ohm}$, the VSWR is equal to 2 as shown by simulation at Figure 2(b), and for a load $200 \mathrm{ohm}$ the VSWR goes to 4 . This confirms that when the load changes and it differs from the line's characteristic impedance $Z_{0}$, VSWR also changes because of reflected waves $[7,8]$.

The second scenario, as in Figure 3, considers a gateway, three transmission lines, with $Z_{0}$ impedance equal to $50 \mathrm{ohm}$, a slotted line and a load of $50 \mathrm{ohm}$. In this case the transmission line sizes are fixed and the parameter that varies is the dimension of the slotted guide. The slotted guide is moved in different positions. Simulations are performed at frequency of $3 \mathrm{GHz}$. From simulation, as shown in Figure 3 the value of VSWR is equal to 1, and this represents the best case where the reflected waves are too low, closed to zero.

The third scenario, presented in Figure 4, considers a gateway, three transmission lines, with $Z_{0}$ impedance equal to $50 \mathrm{ohm}$, a slotted line and a load of 0 (zero) ohm or the short circuit. In this case the transmission line sizes are fixed. The parameter that varies is dimension of slotted guide. The slotted guide is moved in different position in order to get a VSWR value as shown in Figure 4. Simulation is performed at frequency of $3 \mathrm{GHz}$. At this case, the value of VSWR is equal to 9.68, confirming too high level of reflected waves and indicating that an improvement of VSWR should be considered.

\section{VSWR Experimental Measurements}

The laboratory scheme used for VSWR measurement in Figure 5 is given [9-12]. Transmitter generates intermediate frequency which can be selected as one of the four different frequency separated channels, with frequency separation of $27 \mathrm{MHz}$. The power level is adjustable from $0 \mathrm{~dB}$ to $-25 \mathrm{~dB}$.

The transmitter uses two switches, SW1 and SW2, for channel frequency selection, as presented in Table $\mathbf{1 .}$ 


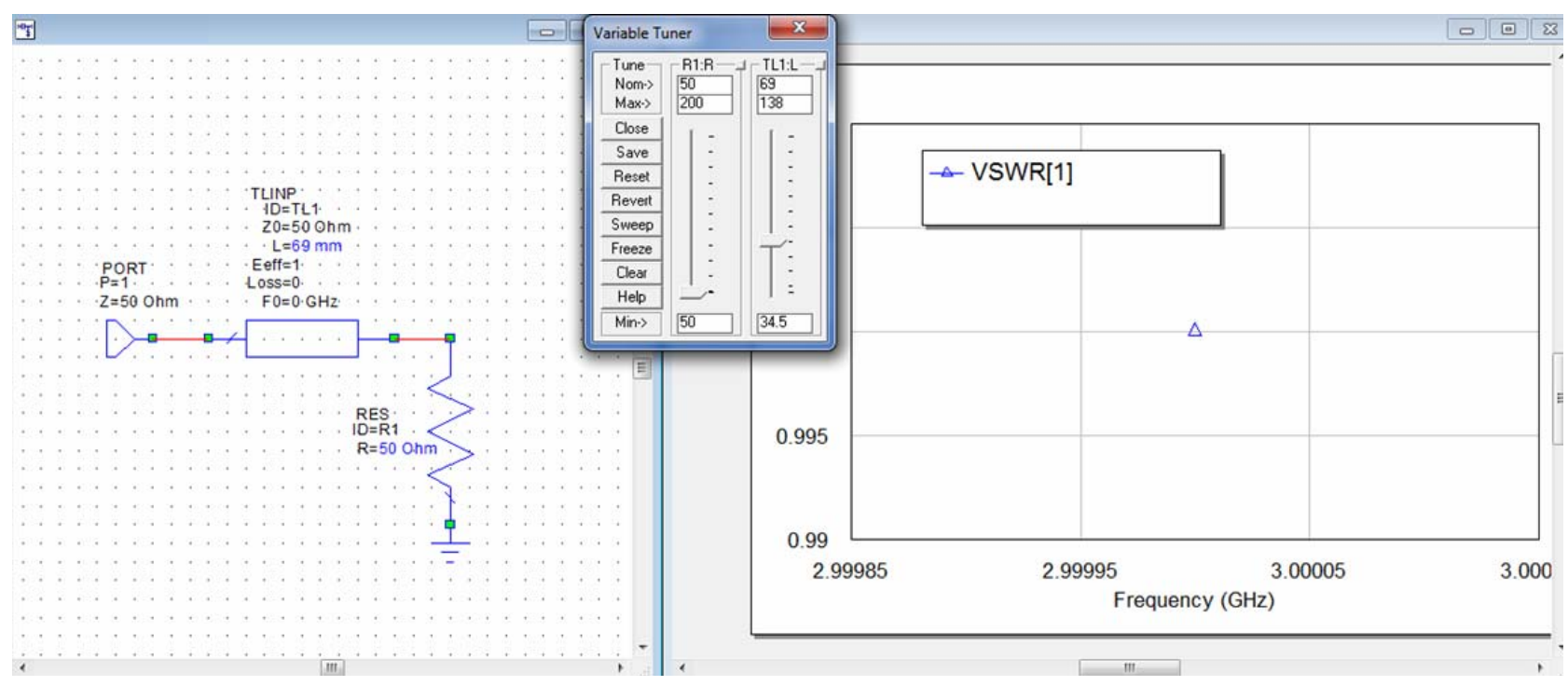

(a)

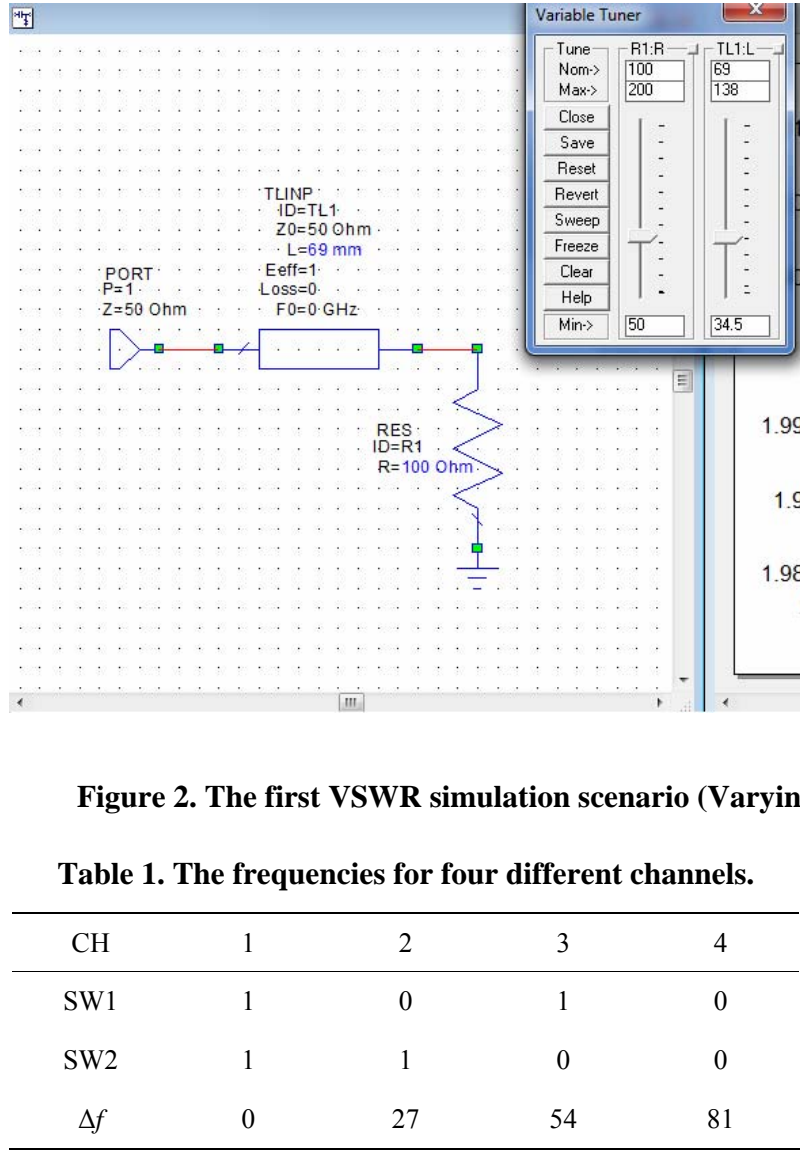

Frequency is determined as:

$$
f_{I F}=2400+\Delta f(\mathrm{MHz})
$$

Intermediate frequency signal, which comes from the transmitter, enters to the up converter via a coaxial cable. Up converter is presented in Figure 6.

The up converter has a local oscillator of frequency $f_{L O}$

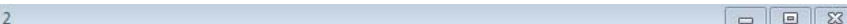

load). (a) Case for $50 \mathrm{ohm}$ load; (b) Case for $100 \mathrm{ohm}$ load.

$=8350 \mathrm{MHz}$, which is synthesized with PLL, while generating signals at the output of the mixer. After mixing with intermediate frequency, is generated signal at radiofrequency band as:

$$
f_{R F}=f_{I F}+f_{L O}
$$

For four known intermediate frequencies and local oscillator frequency, RF frequencies are given in Table 2.

As up converter output, the RF signal, through a coaxial cable, enters to a waveguide coupling elements as in Figure 7. Waveguide elements, from left to right are: coax waveguide adapter, waveguide $6 \mathrm{~cm}$, waveguide 15 $\mathrm{cm}$, slotted line with RF output, and a waveguide of $6 \mathrm{~cm}$ dedicated for load connection. 

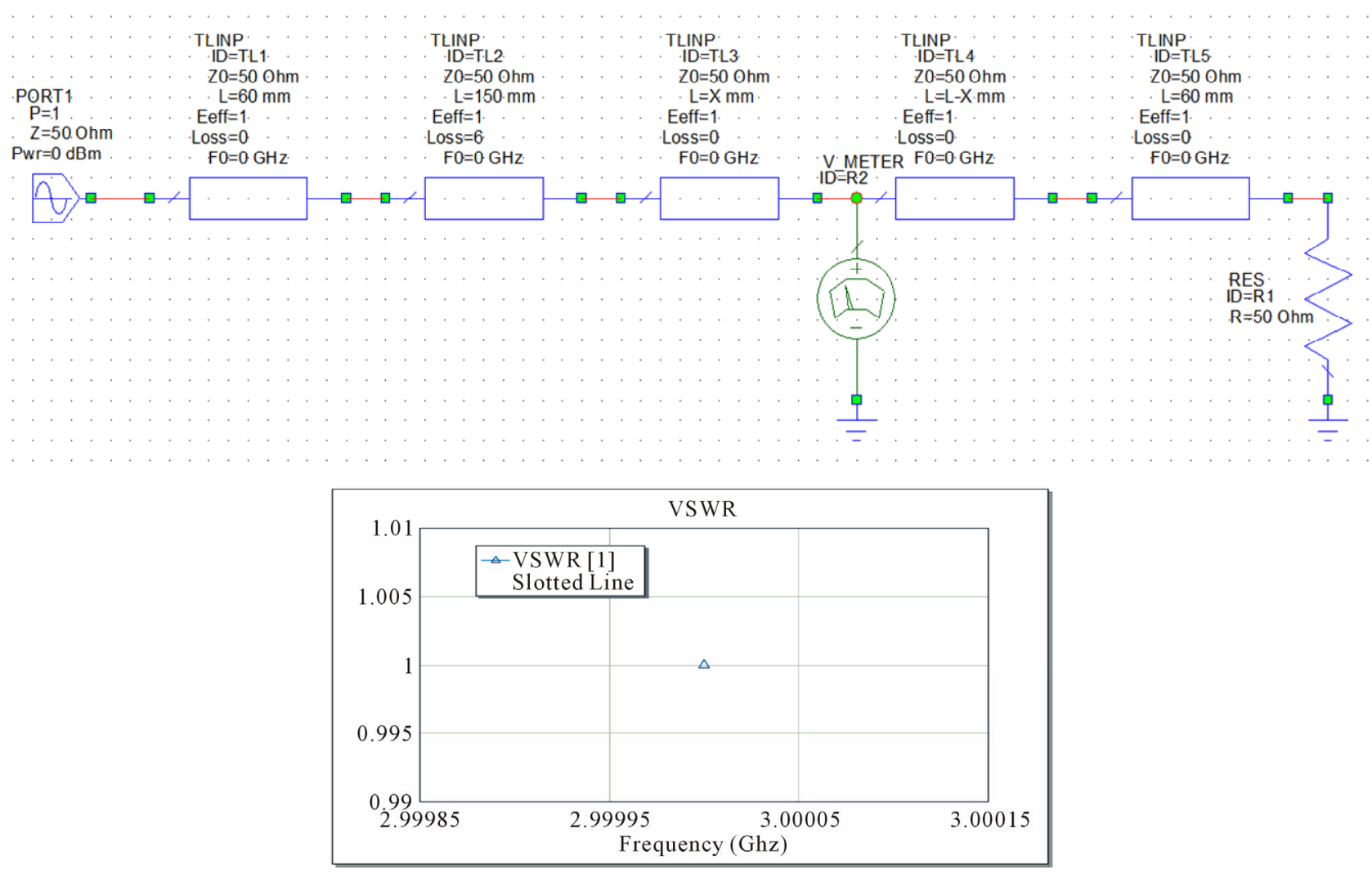

Figure 3. The second VSWR simulation scenario (Matched load).
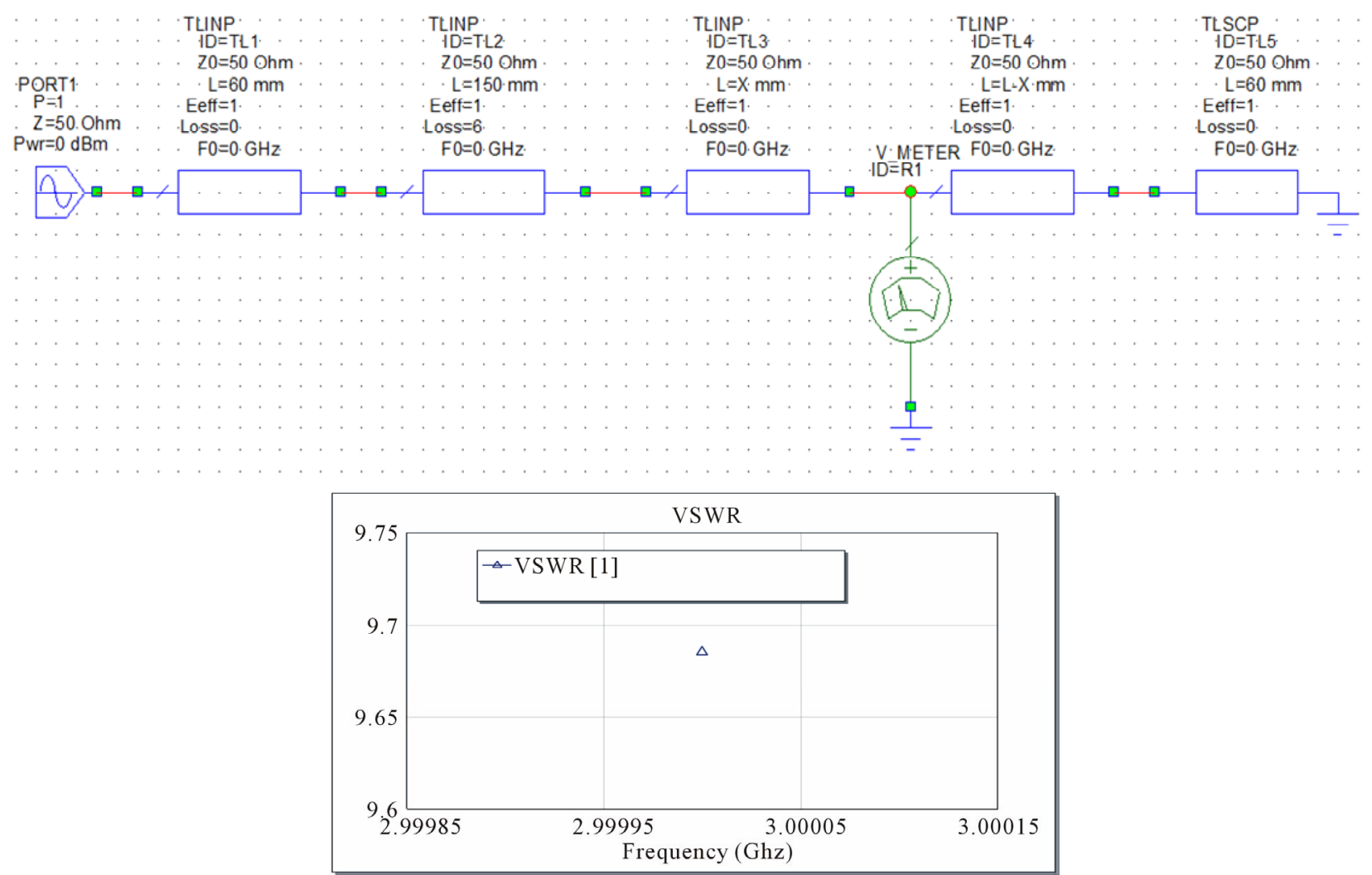

Figure 4. The third VSWR simulation scenario (Short circuit load). 


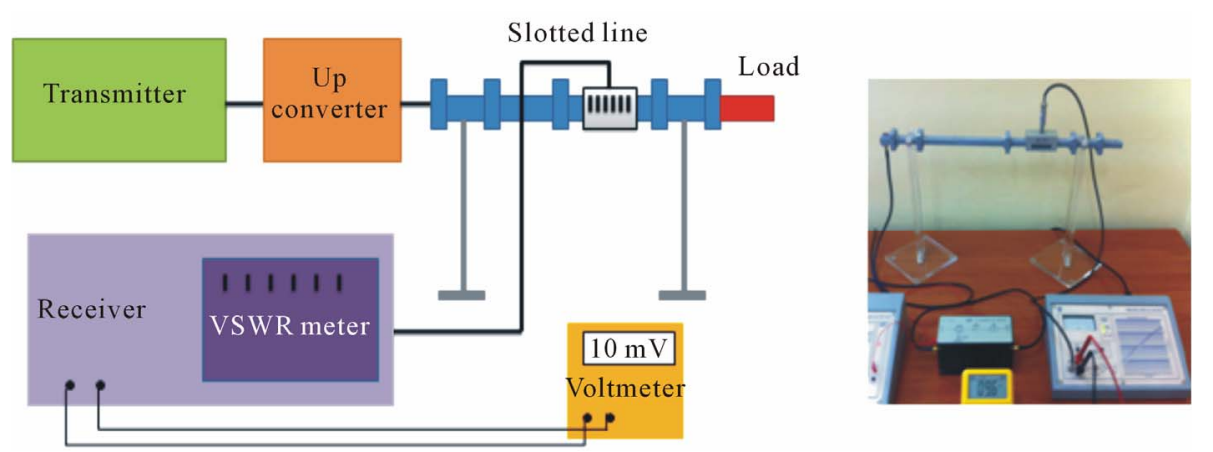

Figure 5. The VSWR measurement scheme.

RF signal, from slotted line, through a coaxial cable, enters the receiver as in Figure 8.

VSWR measurements were carried out to intermediate frequency, $f_{I F}=2400 \mathrm{MHz}, \Delta f=0$. This means that the signal at the receiver, after exiting the slotted line, is at the first frequency channel, equal to $10.750 \mathrm{MHz}$. Measurements are performed for a signal level at the transmitter equal to $-7.5 \mathrm{~dB}$ and by moving the slotted line position in order to measure the maximum value and minimum value of voltage in the receiver. Measuring the maximum and minimum voltage values and applying Equation (3), the VSWR can be calculated. Measurements are performed for these three cases:

- $50 \mathrm{ohm}$ load.

- Short circuit load.

- Horn antenna.

\subsection{Measurement for $50 \mathrm{ohm} \mathrm{Load}$}

In case the load of $50 \mathrm{ohm}$ is connected, as in Figure 9, the measurement results are presented in Table 3.

From measurements it is:

$$
V_{\max }=10.8 \mathrm{mV} \text { and } V_{\min }=9.6 \mathrm{mV}
$$

Applying Equation (3), for WSWR yields out:

$$
\text { VSWR }=1.125
$$

This VSWR results that even if the load connected (50 ohm) is the same with the line's impedance, the VSWR is not equal to one but it is close to that theoretical value. The reason behind for this difference lies on connections used between wave guides.

\subsection{Measurements for a Short Circuit Load}

In case a short circuit is loaded, as in Figure 10, the measurement results are presented in Table 4.

From measurements it is:

$$
V_{\max }=11.3 \mathrm{mV} \text { and } V_{\min }=1.6 \mathrm{mV} \text {. }
$$

Applying Equation (3), for WSWR yields out:

$$
\text { VSWR }=7.06
$$

For a short circuit loaded the value of VSWR is too

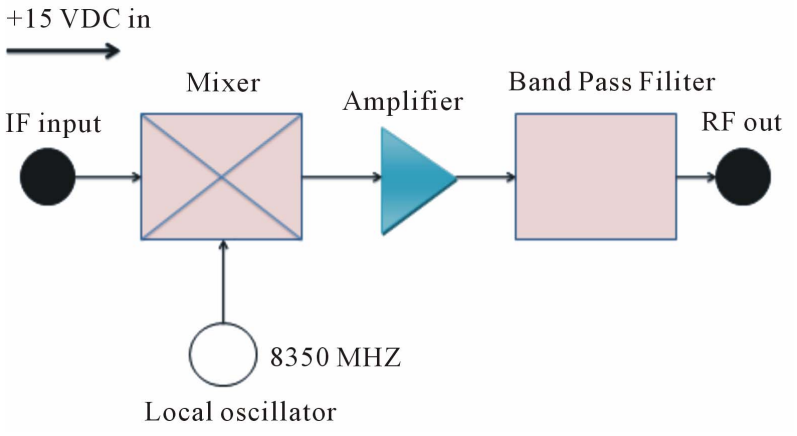

Figure 6. Up converter.

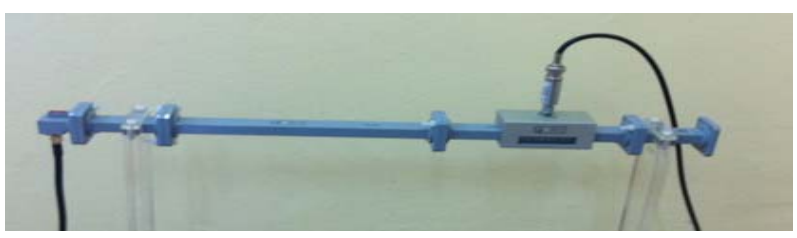

Figure 7. Waveguide elements.

Table 2. RF frequencies based on the selected channel.

\begin{tabular}{ccccc}
\hline $\mathrm{CH}$ & 1 & 2 & 3 & 4 \\
\hline$f_{R F}(\mathrm{MHz})$ & 10750 & 10777 & 10804 & 10831 \\
\hline
\end{tabular}

Table 3. Measurements for a $50 \mathrm{ohm}$.

\begin{tabular}{cc}
\hline "Slotted Guide" position & Voltage value in the receiver \\
\hline $1 \mathrm{~cm}$ & $9.9 \mathrm{mV}$ \\
$1.5 \mathrm{~cm}$ & $10.2 \mathrm{mV}$ \\
$2 \mathrm{~cm}$ & $10.8 \mathrm{mV}$ \\
$2.5 \mathrm{~cm}$ & $10.5 \mathrm{mV}$ \\
$3 \mathrm{~cm}$ & $9.6 \mathrm{mV}$ \\
$3.5 \mathrm{~cm}$ & $9.6 \mathrm{mV}$ \\
$4 \mathrm{~cm}$ & $10.7 \mathrm{mV}$ \\
$4.5 \mathrm{~cm}$ & $10.7 \mathrm{mV}$ \\
$5 \mathrm{~cm}$ & $10.2 \mathrm{mV}$ \\
\hline
\end{tabular}




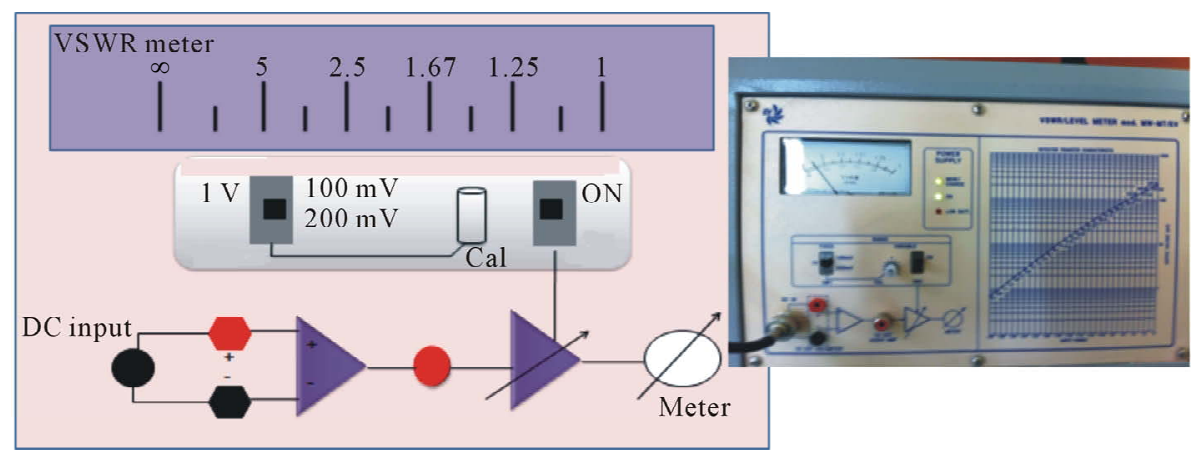

Figure 8. The receiver with VSWR meter.

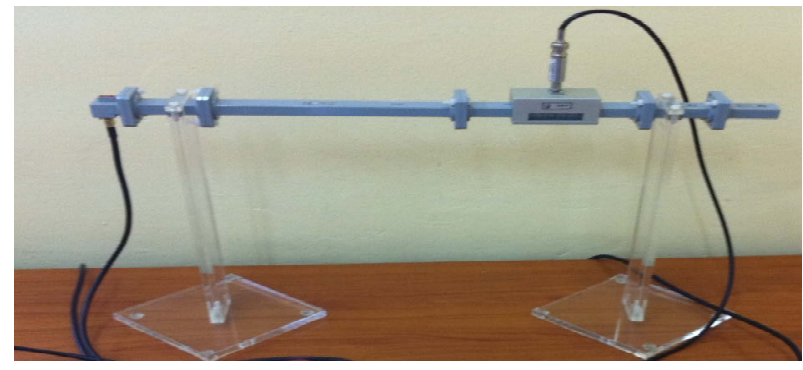

Figure 9. A $50 \mathrm{ohm}$ load connected.

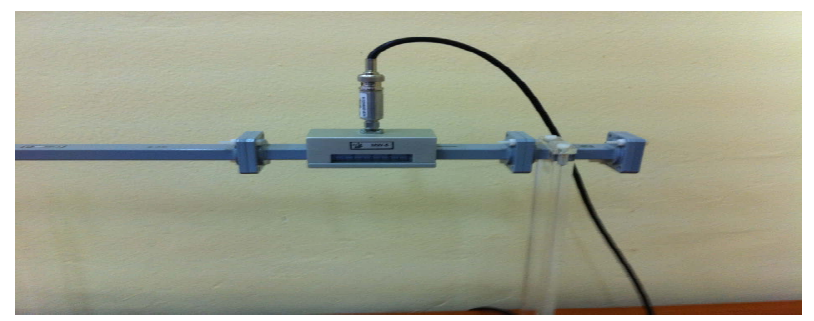

Figure 10. A short circuit connected.

Table 4. Measurements for a short circuit load.

\begin{tabular}{cc}
\hline "Slotted Guide" position & Voltage value in the receiver \\
\hline $1 \mathrm{~cm}$ & $10.9 \mathrm{mV}$ \\
$1.5 \mathrm{~cm}$ & $4.5 \mathrm{mV}$ \\
$2 \mathrm{~cm}$ & $1.8 \mathrm{mV}$ \\
$2.5 \mathrm{~cm}$ & $8.8 \mathrm{mV}$ \\
$3 \mathrm{~cm}$ & $10.6 \mathrm{mV}$ \\
$3.5 \mathrm{~cm}$ & $5.3 \mathrm{mV}$ \\
$4 \mathrm{~cm}$ & $1.6 \mathrm{mV}$ \\
$4.5 \mathrm{~cm}$ & $8.3 \mathrm{mV}$ \\
$5 \mathrm{~cm}$ & $11.3 \mathrm{mV}$ \\
\hline
\end{tabular}

high and losses as well.

\subsection{Measurements for a Horn Antenna Loaded}

In case a horn antenna with a gain of $15 \mathrm{~dB}$, as a load

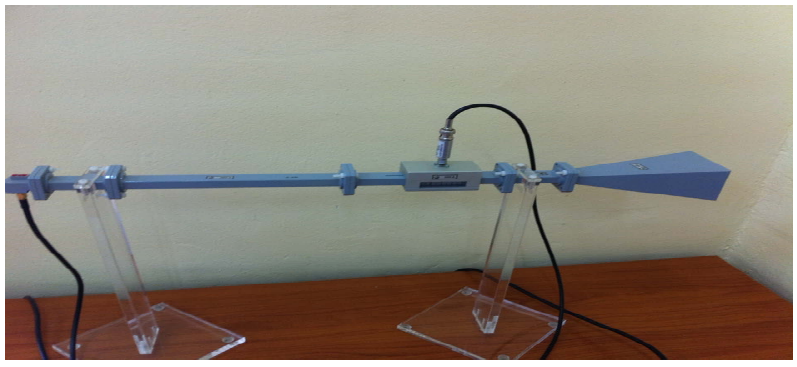

Figure 11. A horn antenna (15 dB) connected.

Table 5. Measurements for a horn antenna.

\begin{tabular}{cc}
\hline "Slotted Guide" position & Voltage value in the receiver \\
\hline $1 \mathrm{~cm}$ & $10.8 \mathrm{mV}$ \\
$1.5 \mathrm{~cm}$ & $10.1 \mathrm{mV}$ \\
$2 \mathrm{~cm}$ & $9.1 \mathrm{mV}$ \\
$2.5 \mathrm{~cm}$ & $10.1 \mathrm{mV}$ \\
$3 \mathrm{~cm}$ & $10 \mathrm{mV}$ \\
$3.5 \mathrm{~cm}$ & $9.4 \mathrm{mV}$ \\
$4 \mathrm{~cm}$ & $9.3 \mathrm{mV}$ \\
$4.5 \mathrm{~cm}$ & $9.9 \mathrm{mV}$ \\
$5 \mathrm{~cm}$ & $10.7 \mathrm{mV}$ \\
\hline
\end{tabular}

connected, as in Figure 11 the measurement results in Table 5 are given.

From measurements it is:

$$
V_{\max }=10.8 \mathrm{mV} \text { and } V_{\min }=9.1 \mathrm{mV} \text {. }
$$

Applying Equation (3), for WSWR yields out:

$$
\text { VSWR }=1.18
$$

For a horn antenna as a load connected, the value of VSWR is relatively small, indicating a good matching between communication elements.

\section{Conclusions}

Evaluation of the VSWR on microwave circuits and lines 
is very important. Determining the value of VSWR allows concluding about the efficiency of transmission and receiver systems. This conclusion is relevant related to transmission or receiver circuits needs to be improved. Both, results from simulations and from experimental measurements indicate that, the difference between the line's characteristic impedance and the load value connected to it creates different VSWR values. The higher difference between line's impedance and the load value connected to it generates the higher VSWR, consequently the lower efficiency of the communication system.

There is a difference between VSWR values generated by simulations and experimental results, since during simulations, the losses due to connection between elements, are not considered. In some cases when VSWR needs to be reduced in order to increase the efficiency of transmission lines, adapter circuits should be used. The adapter circuit can be installed between the transmitter and the antenna feed line. This method will allow transmitters to operate with a lower VSWR.

\section{REFERENCES}

[1] A. Pekka, "Microwave Transmission-Line Networks for Backward-Wave Media and Reduction of Scattering," TKK Radio Science and Engineering Publications, 2009.

[2] J. D. Kraus and R. J. Marhefka, “Antennas for All Applications," 3rd Edition, McGraw Hill, New York, 2003.

[3] C. A. Balanis, "Antenna Theory Analysis and Design," 2nd Edition, John Wiley \& Sons, Inc., New York, 1997.

[4] Y. T. Lo and S. W. Lee, "Antenna Handbook, Theory,
Applications, and Design," Van Nostrand Reinhold, New York, 1988.

[5] W. Darrin, "Understanding SWR by Example," Simulations, QST (C) ARRL, 2006.

[6] T. G. Spence, D. H. Werner and R. D. Groff, "Genetic Algorithm and Optimization of Some Novel Broadband and Multiband Microstrip Antennas," The Department of Electrical Engineering, Pennsylvania State University, University Park, 2004.

[7] G. Bindu, A. Lonappan, V. Thomas, C. K. Aanandan and K. T. Mathew, "Active Microwave Imaging for Breast Cancer Detection," Progress in Electromagnetics Research, Vol. 58, 2006, pp. 149-169. doi:10.2528/PIER05081802

[8] V. Vountesmeri and J. K. Rodriguez, "Magnetoresistive Power Sensor for Measurement in Situ of RF Power Absorbed by Tissue," IEEE Transactions on Instrumentation and Measurement, Vol. 49, No. 3, 2000, pp. 513-516. doi:10.1109/19.850386

[9] M. Dogan and F. Ustuner, "A Telemetry Antenna System for Unmanned Air Vehicles," Progress in Electromagnetics Research Symposium Proceedings, Cambridge, 5 July 2010.

[10] Jiri Polivka Spacek Labs, "Inc. High Frequency Electronics," Summit Technical Media, LLC, 2007.

[11] P. O. Otasowie and E. A. Ogujor, "Voltage Standing Wave Ratio Measurement and Prediction," International of Physical Sciences, Vol. 4, No. 11, 2009, pp. 651-656.

[12] Zh. Yang, et al., "A Simple and Accurate Measurement Method for WSVR of Waveguide to Coaxial Transitions," International Conference on Measuring Technology and Mechanotronics Automation, Vol. 1, 2010, pp. 339-341. 